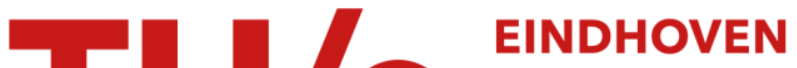

\section{The Share-a-Ride problem with stochastic travel times and stochastic delivery locations}

\section{Citation for published version (APA):}

Li, B., Krushinsky, D., van Woensel, T., \& Reijers, H. A. (2016). The Share-a-Ride problem with stochastic travel times and stochastic delivery locations. Transportation Research. Part C: Emerging Technologies, 67, 95-108. https://doi.org/10.1016/j.trc.2016.01.014

\section{Document license:}

TAVERNE

DOI:

10.1016/j.trc.2016.01.014

Document status and date:

Published: 01/06/2016

\section{Document Version:}

Publisher's PDF, also known as Version of Record (includes final page, issue and volume numbers)

\section{Please check the document version of this publication:}

- A submitted manuscript is the version of the article upon submission and before peer-review. There can be important differences between the submitted version and the official published version of record. People interested in the research are advised to contact the author for the final version of the publication, or visit the $\mathrm{DOI}$ to the publisher's website.

- The final author version and the galley proof are versions of the publication after peer review.

- The final published version features the final layout of the paper including the volume, issue and page numbers.

Link to publication

\section{General rights}

Copyright and moral rights for the publications made accessible in the public portal are retained by the authors and/or other copyright owners and it is a condition of accessing publications that users recognise and abide by the legal requirements associated with these rights.

- Users may download and print one copy of any publication from the public portal for the purpose of private study or research.

- You may not further distribute the material or use it for any profit-making activity or commercial gain

- You may freely distribute the URL identifying the publication in the public portal.

If the publication is distributed under the terms of Article 25fa of the Dutch Copyright Act, indicated by the "Taverne" license above, please follow below link for the End User Agreement:

www.tue.nl/taverne

Take down policy

If you believe that this document breaches copyright please contact us at:

openaccess@tue.nl

providing details and we will investigate your claim. 


\title{
The Share-a-Ride problem with stochastic travel times and stochastic delivery locations
}

\author{
Baoxiang $\mathrm{Li}^{\mathrm{a}, *}$, Dmitry Krushinsky ${ }^{\mathrm{a}}$, Tom Van Woensel ${ }^{\mathrm{a}}$, Hajo A. Reijers ${ }^{\mathrm{b}, \mathrm{c}}$ \\ ${ }^{\mathrm{a}}$ Department of Industrial Engineering and Innovation Sciences, Eindhoven University of Technology, Eindhoven, The Netherlands \\ ${ }^{\mathrm{b}}$ Department of Computer Science, VU University Amsterdam, Amsterdam, The Netherlands \\ ${ }^{\mathrm{c}}$ Department of Mathematics and Computer Science, Eindhoven University of Technology, Eindhoven, The Netherlands
}

\section{A R T I C L E I N F O}

\section{Article history:}

Received 14 June 2015

Received in revised form 18 January 2016

Accepted 18 January 2016

Available online 27 February 2016

\section{Keywords:}

Share-a-Ride problems

Adaptive large neighborhood search

Stochastic travel times

Stochastic delivery locations

Sampling strategies

\begin{abstract}
A B S T R A C T
We consider two stochastic variants of the Share-a-Ride problem: one with stochastic travel times and one with stochastic delivery locations. Both variants are formulated as a two-stage stochastic programming model with recourse. The objective is to maximize the expected profit of serving a set of passengers and parcels using a set of homogeneous vehicles. Our solution methodology integrates an adaptive large neighborhood search heuristic and three sampling strategies for the scenario generation (fixed sample size sampling, sample average approximation, and sequential sampling procedure). A computational study is carried out to compare the proposed approaches. The results show that the convergence rate depends on the source of stochasticity in the problem: stochastic delivery locations converge faster than stochastic travel times according to the numerical test. The sample average approximation and the sequential sampling procedure show a similar performance. The performance of the fixed sample size sampling is better compared to the other two approaches. The results suggest that the stochastic information is valuable in real-life and can dramatically improve the performance of a taxi sharing system, compared to deterministic solutions.
\end{abstract}

(c) 2016 Elsevier Ltd. All rights reserved.

\section{Introduction}

People and freight transportation operations are handled separately, both in the academic literature and in practice. Combining people and freight flows creates attractive business opportunities because the same transportation needs can be met with less vehicles and drivers. Moreover, while cities become more crowded and polluted, sharing transportation network can alleviate urban congestion and reduce environmental pollution. For instance, Fagnant and Kockelman (2014) described a shared autonomous vehicle operation which applying agent-based model, the results shows the estimated significant environmental benefits of shared vehicles, comparing to conventional vehicle ownership.

We elaborate a mixed commodity Share-a-Ride services in this paper, where people and parcels are simultaneously managed by the same network, as described in Li et al. (2014). It involves planning the taxi routes capable of accommodating people and freight as much as possible under a given set of constraints related to pickup and delivery times, the capacity of a taxi, etc. The application can be extended to other transportation modes, such as bus, train or tram services.

One important issue is that the deterministic Share-a-Ride Problem (SARP) described in Li et al. (2014) did not adequately cover real-life situations. For example, it ignores road congestion, and the information of requests may not always be known beforehand. This motivated us to consider a stochastic variant of the SARP. In this paper, two stochastic variants of the SARP

\footnotetext{
* Corresponding author at: Paviljoen E18, Den Dolech 2, Eindhoven University of Technology, 5612 AZ Eindhoven, The Netherlands. Tel.: +31 (0)402472693.

E-mail address: B.li@tue.nl (B. Li).
} 
(stochastic travel times and delivery locations) are studied. The stochastic travel times is affected by the traffic conditions. Normally, the stochastic location problem happens in the rural area and arises recently. We illustrate a situation that for requests in the rural area, the exact location is not known beforehand, only the closest central address is known. For example, most part of the countryside of China, the exact address for every house does not exist. The parcel delivery request for the rural area is fulfilled in the following ways: (1) deliver to a central area or landmark address, and wait the customer to pick up and (2) get the exact location by phone call when the driver reaches near the central address. The passenger request is served by the passenger's navigation to the precise address when almost arrive the approximate location.

The remainder of this paper is organized as follows. The literature is reviewed in Section 2. Section 3 briefly introduces the two-stage recourse model formulations for the stochastic SARP. The first stage deals with the deterministic side of the problem, and the second stage incorporates stochasticity via scenarios. In Section 4, the proposed metaheuristic and three sampling methods (fixed sample size sampling, sample average approximation and sequential sampling procedure) are presented. The computational results are presented and analyzed in Section 5. Finally, Section 6 concludes the paper with a summary and conclusions.

\section{Literature review}

The description of the static SARP was proposed recently. Li et al. (2014) explained the conceptual and mathematical models in which people and parcels are handled by the same network. A heuristic based on the adaptive large neighborhood search was proposed by Li et al. (2016) to solve the SARP static efficiently. As mentioned in Li et al. (2014), the SARP could be casted in the branch of Dial-a-Ride Problem (DARP). The difference between the DARP and the SARP is that the SARP introduces different requirements and objectives into the DARP based on people and parcels sharing the same vehicles. Besides the static DARP, we mainly considered stochastic DARP in this paper. Stochastic DARP addressed in the literature with various sources of stochasticity: stochastic customer delays, stochastic travel times, and stochastic demands, see e.g. Fu (2002) and Heilporn et al. (2011).

Fu (2002) discussed a study on the Dial-a-Ride paratransit scheduling problems that are subject to time-varying stochastic traffic congestion. The author explicitly modeled time-dependent stochastic travel times in the problem formulation. The conventional heuristic algorithms were extended for solving the proposed problem. Heilporn et al. (2011) proposed a stochastic DARP with stochastic customer delays: if a customer is absent when the vehicle serves the pickup location, the request is fulfilled by an alternative service whose cost is added. This problem was solved by an integer L-shaped algorithm. Schilde et al. (2011) analyzed a dynamic stochastic Dial-a-Ride problem with expected return transports; some requests are dynamic and stochastic. Four different modifications of metaheuristic solution approaches were tested for this problem: dynamic versions of variable neighborhood search (VNS), stochastic VNS (S-VNS), modified versions of the multiple plan approach (MPA) and the multiple scenario approach (MSA). Hu et al. (2015) investigated a closed-loop vehicle routing problem with pickup and delivery (VRPPD, similar to DARP), with uncertain pickup and deterministic delivery. A VNSbased algorithm was developed, and uncertainty of demand was treated by two rounds of routing and a dynamic pickup strategy: first, a priori routes were generated by solving a VRPPD whose pickup demands were estimated; second, the routes were generated to meet the unmet demands.

According to King and Wallace (2012), scenario analysis is an essential part of a stochastic problem. The most common method is a sample average approximation (SAA), which is a Monte Carlo simulation-based approach to stochastic discrete optimization problems (Kleywegt et al., 2001). Bayraksan and Morton (2011) proposed a sequential sampling procedure (SSP) method, and they assume that a sequence of feasible solutions with an optimal limit point is given as input to the procedure. Such a sequence can be generated by solving a series of sampling problems with increasing sample size, or it can be found by any other viable method.

The issue of scenario analysis is that if the scenario tree is big, it is easy to converge to a relatively good solution. However, handling large scenario trees is time-consuming. Therefore, efficient scenario tree reduction algorithms were developed to reduce the size of the scenario tree. For instance, the algorithm developed by Dupačová et al. (2003) efficiently reduces the scenario tree without losing the accuracy. Accord to the computation of the given instances, after $50 \%$ reduction of the scenario tree, the optimally reduced tree still had about $90 \%$ relative accuracy.

When it comes to the methodology part, both exact and heuristic methods can be used to solve stochastic problems. Exact method includes the branch and price (Christiansen and Lysgaard, 2007), the L-shaped method (Heilporn et al., 2011), the branch and cut (Gauvin et al., 2014), and so on. For the heuristic method, there are two survey papers related to metaheuristics that are currently being applied to optimization under uncertainty: Bianchi et al. (2006) presented one survey on metaheuristics for stochastic combinatorial optimizations; here, special attention is given to the hybrid of heuristic and exact methods. Moreover, Gutjahr (2011) presented another survey as an addendum to the previous paper (Bianchi et al., 2006). Typical methodology includes insertion heuristic, tabu search, genetic algorithms Marković et al. (2015). The exact method is good at solution quality, but cannot solve the real-life problem. By applying the heuristic method, one can quickly find a good feasible solution, though the optimality is not guaranteed. Other methodology includes hybrid metaheuristics approach (Bianchi et al., 2006), rolling horizon mechanism (Wang et al., 2014) and so on.

In all the heuristic, neighborhood search heuristics are typical methods to solve the DARP, e.g. Cordeau and Laporte (2003) described a tabu search heuristic, Ropke and Pisinger (2006) presented an adaptive large neighborhood search heuristic, Gendreau et al. (2006) proposed neighborhood search heuristic. Muelas et al. (2015) presented a distributed VNS algorithm 
and tested on large-scale problem (up to 16,000 requests or 32,000 locations). The neighborhood search based algorithm proved to be efficient to solve high dimensional dial-a-ride problems. Therefore, the methodology we choose for this paper is based on neighborhood search heuristics. The contribution of this paper is that we define two variants of the Share-a-Ride problem with stochastic travel times and stochastic delivery locations. We provide the first application of the neighborhood search heuristic to the stochastic version of the problem. Further, we compare different solution methodologies, which integrate an adaptive large neighborhood search (ALNS) algorithm and different sampling strategies. The comparison between the performance of several approaches to handle stochasticity is discussed, which includes fixed sample size sampling (FSS), sample average approximation (SAA), and sequential sampling procedure (SSP). Finally, we quantify the benefits of incorporating the stochastic information.

\section{The stochastic SARP variants}

As stated, Li et al. (2014) presented the first mathematical formulation for the deterministic SARP. In order to consider the expected cost/profit related to the stochastic SARP, a two-stage recourse model (first proposed by Dantzig (1955) and then implemented widely) will be used. In the two-stage stochastic programming model with recourse, the first stage determines the vehicle visiting plan before the stochastic travel times or exact delivery locations for parcels are realized. Once these random variables are known, it is possible to check whether the time window and ride time constraints are satisfied. Thus, the profit, which is uncertain in the first stage, can be calculated, and the expected penalty cost is considered in the second stage.

Following the notations of Li et al. (2014) (as shown in Table 1), let $\sigma$ denote the number of requests to be served, which include $m$ parcels and $n$ passengers. The SARP is defined on a complete undirected graph $\mathcal{G}=(V, E)$ where $V=V^{p} \cup V^{f} \cup\{0,2 \sigma+1\}$. Subsets $V^{p}$ and $V^{f}$ correspond to passenger and parcel stops, respectively, while stops 0 and $2 \sigma+1$ represent the origin and destination depots of the taxis. For easy referencing, we arrange all stops in $V$ in such a way that all origins precede all destinations. Each request includes one origin and one destination stop. Each stop $i$ is associated with a load $q_{i}$ such that $q_{0}=q_{2 \sigma+1}=0, q_{i}=-q_{i+\sigma}(i=1,2, \ldots, \sigma$, where $i$ denotes origin and $i+\sigma$ denotes destination of a request). Furthermore, the destination of each request can be obtained as its origin offset by a fixed constant $\sigma$. Let $K$ be the set of vehicles. Each vehicle $k \in K$ has a capacity $Q_{k}$ and the total duration of its route cannot exceed $T_{k}$. A time window $\left[e_{i}, l_{i}\right]$ is also associated with node $i \in V$ where $e_{i}$ and $l_{i}$ represent the earliest and latest time, respectively. For each stop $i$, the taxi needs to wait if arrive early than $e_{i}$, and penalty will be caused when the taxi excess the $L_{i}$. Variables $s_{i}$ denote the service time at stop $i$. Distance $d_{i j}$ and travel time $t_{i j}$ are assigned to each edge $(i, j) \in E$.

For each arc $(i, j) \in A$ and each vehicle $k \in K$, let $x_{i j}^{k}=1$ if vehicle $k$ travels from node $i$ directly to node $j$. For each node $i \in V$ and each vehicle $k \in K$, let $\tau_{i}^{k}$ be the time that vehicle $k$ begins to serve node $i$, and $w_{i}^{k}$ be the load of vehicle $k$ after visiting

Table 1

Parameters and variables for the stochastic SARP models.

\begin{tabular}{|c|c|}
\hline$n, m$ & Number of passengers and parcels, respectively \\
\hline K & Set of taxis, $K=\{1,2, \ldots,|K|\}$ \\
\hline$V^{p}$ & Set of passenger stops \\
\hline$V^{f}$ & Set of parcel (freight) stops \\
\hline$V^{p} \cup V^{f}$ & Set of all stops, $V^{p} \cup V^{f}=\{1,2, \ldots, 2 \sigma\}, \sigma=m+n$ \\
\hline V & $=V^{p} \cup V^{f} \cup\{0,2 \sigma+1\}, 0$ and $2 \sigma+1$ represent the origin and the destination of a taxi (i.e., depots), respectively \\
\hline$V^{p, o}$ & Set of passenger origins $V^{p, o}=\{1,2, \ldots, n\}$ \\
\hline$V^{p, d}$ & Set of passenger destinations $V^{p, d}=\{\sigma+1, \sigma+2, \ldots, \sigma+n\}$ \\
\hline$V^{f, o}$ & Set of parcel origins $V^{f, o}=\{n+1, n+2, \ldots, \sigma\}$ \\
\hline$V^{f, d}$ & Set of parcel destinations $V^{f, d}=\{\sigma+n+1, \sigma+n+2, \ldots, 2 \sigma\}$ \\
\hline$q_{i}$ & Weight of request $i$ \\
\hline$\left[e_{i}, l_{i}\right]$ & Time window for request $i$ \\
\hline$Q_{k}$ & Capacity of taxi $k$ \\
\hline$T_{k}$ & Maximum duration of parcel service for taxi $k$ \\
\hline$\eta$ & Maximum number of requests between one passenger service \\
\hline$d_{i j}$ & Distance between stops $i$ and $j$ \\
\hline$t_{i j}$ & Travel time between stops $i$ and $j$ \\
\hline$\alpha$ & Initial fare charged for delivering one passenger \\
\hline$\beta$ & Initial fare charged for delivering one parcel \\
\hline$\gamma_{1}$ & Fare charged for delivering one passenger per kilometer \\
\hline$\gamma_{2}$ & Fare charged for delivering one parcel per kilometer \\
\hline$\gamma_{3}$ & Average cost per kilometer (fuel, tolls, etc.) for delivering requests \\
\hline$\gamma_{4}$ & Discount factor for exceeding the direct delivery time of passengers \\
\hline$C_{d}$ & Penalty incurred if a vehicle arrives later than the upper bound of time window \\
\hline$x_{i j}^{k}$ & Binary decision variables equal to 1 if taxi $k$ goes directly from stop $i$ to stop $j$ \\
\hline$\tau_{i}^{k}$ & Time point when taxi $k$ leaves stop $i$ \\
\hline$r_{i}^{k}$ & Time spent by request $i$ in taxi $k, r_{i}^{k}=\tau_{i+\sigma}^{k}-\tau_{i}^{k}, i \in C$ \\
\hline$w_{i}^{k}$ & Load of taxi $k$ after visiting stop $i$ \\
\hline$P_{i}$ & Index of request $i$ in a service sequence of a taxi \\
\hline
\end{tabular}


node $i$. For each passenger $i\left(i \in V^{p, o}\right)$, let $r_{i}^{k}$ be his/her ride time on vehicle $k$, the maximum ride time of $i$ is denoted by $\varpi_{i}$. The initial profits obtained from a passenger and a parcel are represented by $\alpha$ and $\beta$, respectively; while the average profit per unit distance are denoted by $\gamma_{1}$ and $\gamma_{2}$, the cost per unit distance is $\gamma_{3}$. The discount factor for exceeding the direct delivery time of passengers is represented by $\gamma_{4}$. Let $n$ and $m$ be the number of passengers and parcels, respectively. We use variables $P_{i} \in\{0,1, \ldots, 2(m+n+1)\}$ to define the serving sequence of taxis, and the maximum number of requests that can be served within a trip of one passenger is represented by $\eta$. To ensure the validity of the sequence constraints, we set $M=2(m+n)+1$. Finally, we denote the $\max (0, x)$ by $x^{+}$and random vector as $\xi$.

We intentionally consider the two sources of stochasticity separately (instead of combining them in one model) in order to better understand the implications of each source.

\subsection{Stochastic travel times}

The first stage formulation for the SARP with stochastic travel times can be formulated as follows:

$$
\max _{x}\left\{f(x)=\left[\sum_{i \in V^{p, o}} \sum_{j \in V} \sum_{k \in K}\left(\alpha+\gamma_{1} d_{i, i+\sigma}\right) x_{i j}^{k}+\sum_{i \in V^{f, o}} \sum_{j \in V} \sum_{k \in K}\left(\beta+\gamma_{2} d_{i, i+\sigma}\right) x_{i j}^{k}-\gamma_{3} \sum_{i \in V} \sum_{j \in V} \sum_{k \in K} d_{i j} x_{i j}^{k}\right]+E[Q(x, \xi)]\right\}
$$

where $Q(x, \xi)$ is the optimal value of the second-stage problem, as shown in function (14).

Subject to:

$$
\begin{aligned}
& \sum_{j \in V} \sum_{k \in K} x_{i j}^{k} \leqslant 1, \quad \forall i \in V^{p, o} \cup V^{f, o} \\
& \sum_{i \in V} x_{0, i}^{k}=\sum_{i \in V} x_{i, 2 \sigma+1}^{k}=1, \quad \forall k \in K \\
& \sum_{i \in V} x_{i, 0}^{k}=\sum_{i \in V} x_{2 \sigma+1, i}^{k}=0, \quad \forall k \in K \\
& \sum_{i \in V} x_{i j}^{k}=\sum_{i \in V} x_{i, j+\sigma}^{k}, \quad \forall j \in V^{p, o} \cup V^{f, o}, \quad k \in K \\
& \sum_{j \in V} x_{i j}^{k}=\sum_{j \in V} x_{j i}^{k}, \quad \forall i \in V^{p} \cup V^{f}, \quad k \in K \\
& w_{j}^{k} \geqslant\left(w_{i}^{k}+q_{j}\right) x_{i j}^{k}, \quad \forall i, j \in V, \quad k \in K \\
& w_{i}^{k} \geqslant \max _{0}\left\{, q_{i}\right\}, \quad \forall i \in V, \quad k \in K \\
& \left.w_{i}^{k} \leqslant \min _{k}, Q_{k}, Q_{k}\right\}, \quad \forall i \in V, \quad k \in K \\
& M\left(\sum_{k \in K} x_{i j}^{k}-1\right)+P_{j}-1 \leqslant P_{i}, \quad \forall i, \quad j \in V^{p} \cup V^{f} \\
& M\left(1-\sum_{k \in K} x_{i j}^{k}\right)+P_{j}-1 \geqslant P_{i}, \quad \forall i, \quad j \in V^{p} \cup V^{f} \\
& P_{j+\sigma}-P_{j}-1 \leqslant \eta, \quad \forall j \in V^{p, o} \\
& x_{i j}^{k} \in\{0,1\} ; \quad w_{i}^{k} \in \mathbb{R}_{+} ; \quad P_{i} \in\{0,1, \ldots, 2(m+n+1)\}
\end{aligned}
$$

The objective function (1) maximizes the total profit obtained from people and parcel delivery, and includes three parts: (i) the profit obtained from passengers; (ii) the profit obtained from parcels; and (iii) the cost of the distance traveled.

Constraints (2)-(6) denote that every request can be served at most once (if been visit, should have one preceding and one succeeding stop), and fix the original and destination points of taxis. The capacity and passenger service priority constraints are defined by Constraints (7)-(12). Especially for constraints (10)-(12), they are helps to avoid long detour for the passenger due to parcel delivery. For instance, if we set $\eta$ equal to 0 , no detour for the passenger due to parcel delivery will be allowed. The details about the related constraints can be found in Li et al. (2014).

The second stage model with stochastic travel times is as follows:

$$
Q(x, \xi)=C_{d} \sum_{i \in V} \sum_{k \in K}\left(\tau_{i}^{k}-l_{i}\right)^{+}+\gamma_{4} \sum_{i \in V^{p, o}} \sum_{k \in K}\left(r_{i}^{k} / t_{i, i+\sigma}-1\right)^{+}
$$

Subject to:

$$
\begin{aligned}
& \tau_{j}^{k} \geqslant\left(\tau_{i}^{k}+t_{i j}\right) x_{i j}^{k}, \quad \forall i, j \in V, \quad k \in K \\
& r_{i}^{k}=\tau_{\sigma+i}^{k}-\tau_{i}^{k}, \quad \forall i \in V^{p, o}, \quad k \in K \\
& \tau_{i}^{k} \geqslant e_{i} \quad \forall i, \quad k \in K \\
& \tau_{i}^{k}, \quad r_{i}^{k} \in \mathbb{R}_{+}
\end{aligned}
$$


For the second stage, function (14) sums the expected costs of violating the time windows and ride time constraints. The travel times $r_{i}^{k}$ and $\tau_{i}^{k}$ are stochastic variables affected by $x_{i j}^{k}$ and the random vector $\xi$. Constraints (15) compute the travel times. Constraints (16) define the ride time of requests.

\subsection{Stochastic delivery locations}

The first stage formulation for the SARP with stochastic delivery locations can be formulated as follows:

$$
\max _{x}\left\{f(x)=\left[\sum_{i \in V^{p, o}} \sum_{j \in V} \sum_{k \in K} \alpha x_{i j}^{k}+\sum_{i \in V^{f, o}} \sum_{j \in V} \sum_{k \in K} \beta x_{i j}^{k}\right]+E[Q(x, \xi)]\right\}
$$

Subject to:

$$
(2)-(13)
$$

The objective function (19) maximizes the total profit that can be obtained from people and parcel delivery. $Q(x, \xi)$ in $(19)$ is the optimal value of the second-stage problem, the second stage model with stochastic delivery locations is as follows:

$$
\begin{aligned}
Q(x, \tau, r, \xi)= & \sum_{i \in V} \sum_{k \in K} C_{d}\left(\tau_{i}^{k}-l_{i}\right)^{+}+\gamma_{4} \sum_{i \in V^{p, o}} \sum_{k \in K}\left(r_{i}^{k} / t_{i, i+\sigma}-1\right)^{+}-\gamma_{1} \sum_{i \in V^{p, o}} \sum_{j \in V} \sum_{k \in K} d_{i, i+\sigma} x_{i j}^{k}-\gamma_{2} \sum_{i \in V^{f, o}} \sum_{j \in V} \sum_{k \in K} d_{i, i+\sigma} x_{i j}^{k} \\
& +\gamma_{3} \sum_{i \in V} \sum_{j \in V} \sum_{k \in K} d_{i j} x_{i j}^{k}
\end{aligned}
$$

Subject to:

$$
(15)-(18)
$$

Function (21) calculates the expected costs which include four parts: a penalty for time window violations, a penalty for ride time constraints violations, a cost based on the distance traveled, and (minus) the distance profit obtained for delivering parcels. The distances $d_{i, j}$ and related time parameters become stochastic due to an uncertainty in the location of the parcel delivery points. The travel times $r_{i}^{k}$ and $\tau_{i}^{k}$ are stochastic variables affected by $x_{i j}^{k}$ and the random vector $\xi$.

\section{The ALNS and sampling strategy used for the stochastic SARP}

Researcher can solve deterministic DARP instances with hundreds of requests using heuristic algorithm, for instance, Marković et al. (2015) can solve real-life problem with 450 requests. Li et al. (2014) presented the first mathematical formulation for the deterministic SARP, which can solve instances with 300 requests. In this section, we describe our algorithm based on neighborhood search heuristic present by Li et al. (2014), and integrates a sampling scheme (as shown in Algorithm 1).

Algorithm 1. Algorithm for solving the stochastic SARP.

Input: Initial solution $s$, solution $s_{\text {best }}:=s$;

1 Apply the scenario generation (FSS, SAA or SSP) schema

2 while stopping criteria for scenario generation procedure not reached do

3 while stopping criteria for ALNS not reached do

$4 \quad \mid s^{\prime}:=s$

5 Apply selection operator to select requests for removal

6 Apply perturbation operator to remove selected requests from $s^{\prime}$ and reinsert as many unserved requests as we can into $s^{\prime}$

7 Calculate objective function of $f\left(s^{\prime}\right)$ according to objective function (1) or (19)

8 if $f\left(s^{\prime}\right)>f\left(s_{\text {best }}\right)$ then

$9 \quad \mid s:=s^{\prime}, s_{\text {best }}:=s^{\prime}$

10 else

11 if $f\left(s^{\prime}\right)>f(s)$ then

12

16 end while

Output: $s_{\text {best }}$; 


\subsection{The ALNS for the SARP}

In this subsection, we describe our ALNS heuristic for the SARP. The heuristic used is based on the ALNS described by Li et al. (2016). An initial solution is constructed by assigning every request to a randomly selected vehicle. Next, the ALNS heuristic is used to improve the original solution. In the heuristic, each iteration includes two sub-processes: request selection and perturbation (removal and re-insertion). The probability of accepting a solution follows a simulated annealing scheme, which is the same as in Ropke and Pisinger (2006). Our stopping criterion is 2000 iterations, because no further improvement is observed after around 2000 iterations.

The choice of the selection and perturbation heuristics is governed by a roulette wheel mechanism. We have ten selection operators and seven perturbation operators. By managing the operators via adaptive weights, we diversify the search and find a good balance between the quality of the solution and the running time. For further details, we refer to Li et al. (2016).

\subsection{Sample average approximation}

The SAA method is a sampling-based approach that has been used successfully to solve stochastic programs. According to Kleywegt et al. (2001), M independent batches, each of which has N scenarios are generated in the SAA. The SAA problem is solved $M$ times repeatedly. In each iteration, independently and identically distributed (i.i.d.) random samples $\left\{W^{1}, \ldots, W^{N}\right\}$ are generated for the associated SAA problem. The expectation of the stochastic problem is approximated by the sample average function $\hat{g}_{N}(x):=1 / N \sum_{j=1}^{N} G\left(x, W^{j}\right)$, where $G\left(x, W^{j}\right)$ is a function of two variables $x$ and $W^{j}$. The solution is evaluated by the optimality gap to the "true" solution and the variance of the gap estimator based on the large sample size $N^{\prime}$. If the optimality gap and the variance of the gap estimator are sufficiently small, we accept the solution. For the sequence sampling procedure we refer to Procedure 1 in Appendix A.

\subsection{Sequential sampling procedure}

The second sampling method used is the SSP. According to Bayraksan and Morton (2011), this method is typically applied to solving a sampling problem with an increasing sample size. At iteration $k \geqslant 1$ of the sequential procedure we select a sample size, $n_{k}$, and we use $n_{k}$ total observations to assess the quality of the current solution, $x_{k}$. The quality of this candidate solution is evaluated, which is based on a statistical estimator of the optimality gap of the candidate solution. If the optimality gap estimate falls below a desired value (see Procedure 2 Step 3), then the procedure stops. Otherwise, the procedure continues with a larger sample size. For the proof of asymptotic validity and finite stopping, we refer to Bayraksan and Morton (2011). The differences between the SSP and the SAA are mainly in two aspects: (1) the SSP increases the sampling size in every iteration, the SAA resamples the fixed size of observations in every iteration and (2) the statistical estimator of the optimality gap differs between the SSP and the SAA. For the sequence sampling procedure we refer to Procedure 2 in Appendix A.

\section{Experiments and computational results}

This section presents results of our computational tests sampling. The testing is implemented in JAVA and executed on an Intel Xeon E5-4610 2.4 GHz 6 core CPU 32 GB RAM computer. The purpose of the testing is to compare the FSS, the SAA, and the SSP. The instances are generated based on the Cabspotting database, which records San Francisco's taxi trails in the Bay Area. All instances can be found at http://smartlogisticslab.nl. In this section, we first introduce the instance design for the stochastic SARP model, then report the computational results of the FSS, the SAA, and the SSP. Finally, a comparison between the different approaches is presented.

\subsection{Instance design for the stochastic SARP model}

The instances include 30-75 requests. The number of parcels is twice that of passengers for instances 30_1, 45_1, 60_1, and 75_1, and the number of passengers is two times that of parcels for the other instances. The number of both passengers and parcels are given beforehand. The capacity of each vehicle is 5 units, the weights of passengers and parcels are set to 3 and 1 units, respectively. The time window width for passenger pickup stops is 20 min. The passenger drop-off time is ensured by the travel time constraints (penalty will be added when excess the direct travel time), together with time window varies between 1 to $2 \mathrm{~h}$. Both the time window width for pickup and drop off stops of parcels are within three hours (9:00-12:00). The working time limit of drivers is three hours. The exact destination for parcel requests is not exactly known but is normally distributed around the center position. Distances are calculated in Manhattan metrics $d(x ; y)=\left|x_{1}-y_{1}\right|+\left|x_{2}-y_{2}\right|$, and travel times are calculated as a ratio between the distance traveled and the speed.

Fig. 1 shows a histogram for the distribution of the speed based on 140,862 taxi speed records inside San Fransisco with a passenger inside traveling between 9:00 and 17:00. The average speed equals the distance divided by the travel time. We 


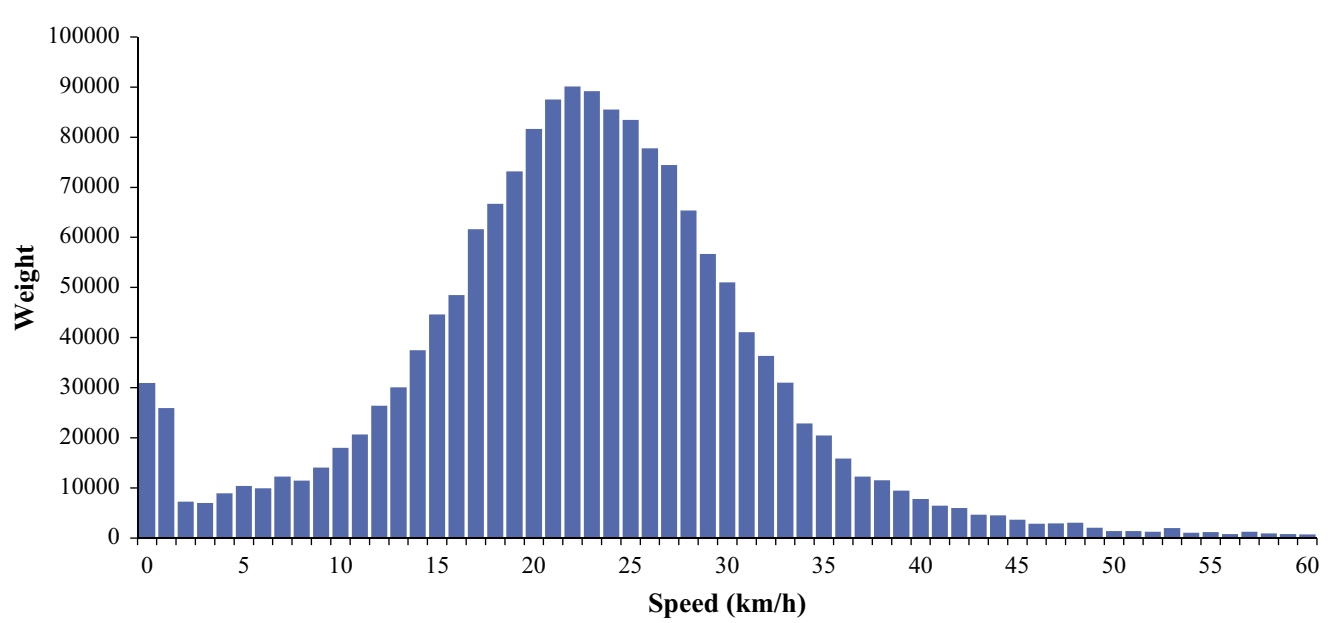

Fig. 1. The distribution of speed between 9:00 and 17:00.

Table 2

List of parameters.

\begin{tabular}{ll}
\hline Descriptions & Values \\
\hline Parameter used for the $S A A$ & 10 \\
$M$, number of the SAA replications & $20,40,60$ \\
$N$, sample size used to calculate the solution & 1000 \\
$N^{\prime}$, sample size used to evaluate the solution & 1.28 \\
$z_{\alpha}$ & 0.10 \\
Parameter used for the SSP & 0.20 \\
$\alpha_{\text {sto }},-\alpha_{\text {sto }}$ is the desired confidence level & $(0.515,0.585,0.740)$ \\
$h^{\prime}$, coefficient of the variance of the solution & $(0.315,0.385,0.540)$ \\
$h$, according to three different initial sample sizes, coefficient of the variance of the solution with $h>h^{\prime}$ & $10^{-7}$ \\
$\delta_{h}\left(h-h^{\prime}\right)$ & $2 \times 10^{-7}$ \\
$\epsilon^{\prime}$, a small positive number that ensures finite stopping & 0.407 \\
$\epsilon$, a small positive number with $\epsilon>\epsilon^{\prime}$ & \\
$p$, parameter that affects the number of samples to generate & \\
\hline
\end{tabular}

present a distance-weighted histogram: each bar reflects the distance driven with the given speed, rather than the number of corresponding trips. Furthermore, we consider the measurements within interval $[0,2]$ as outliers. We choose the speed between any two links from the historical data based on a roulette wheel algorithm: the probability of selecting some speed value is proportional to its weight in the histogram from Fig. 1. The parameters used in the objective function and constraints are defined as the following parameter vector $\left(\alpha, \beta, C_{d}, \gamma_{1}, \gamma_{2}, \gamma_{3}, \gamma_{4}\right)=(3.50,2.33,1.00,2.70$, $0.90,0.60,3.50)$.

The parameters used in the ALNS are listed in Table 2. For the stochastic locations, passengers and parcels delivery locations obey a truncated normal distribution (with a mean value corresponding to some location chosen from the database and a standard deviation of $1 \mathrm{~km}$, truncated at $2 \mathrm{~km}$ ). In the presented results, the negative objective value means that for some scenarios the solution corresponds to a situation when the total cost exceeds the profit.

\subsection{Computational results of the FSS}

Figs. 2 and 3 show the estimated objective values and their standard deviations for different instances. Negative objective values mean that costs and penalties exceed the profit. For stochastic travel times, the solutions keep on improve together with the sample size increases from 10 to 50 . While for stochastic delivery locations, the solutions improve significantly when the sample size increases from 10 to 50 . However, the quality of the generated solutions displays no further improvement if the sample size is larger than 50. Note that the CPU time increases very fast (see Table 3 ). One can see that the objective value varies; the reason is that the travel time depends on the vehicle speed. According to Fig. 1, the speed varies within wide limits, and it becomes difficult to find a solution which is feasible for all such scenarios with different combinations of travel speed. 


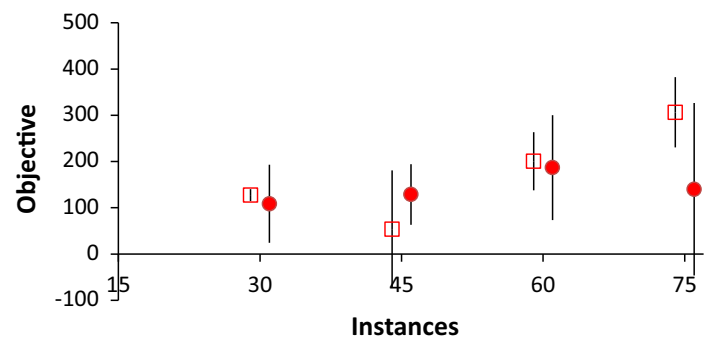

(a) Sample Size 10

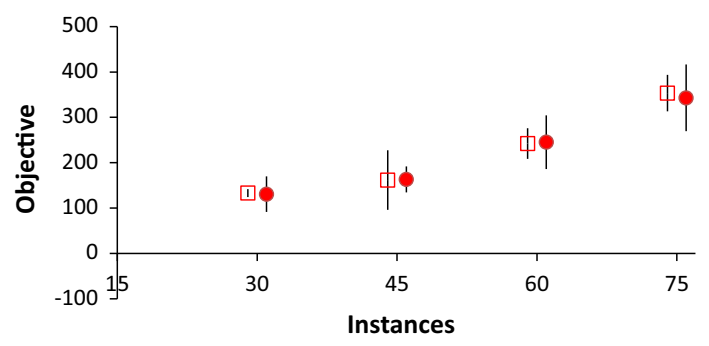

(c) Sample Size 100

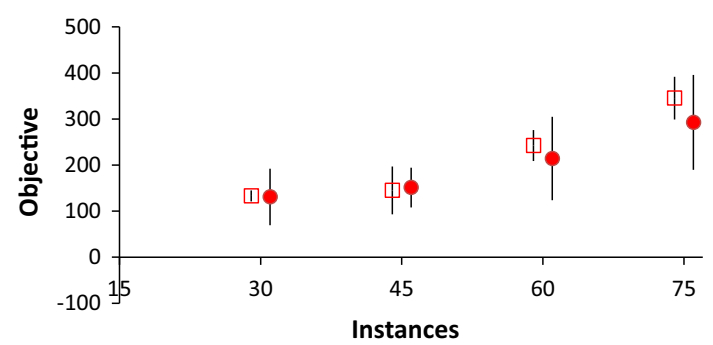

(b) Sample Size 50

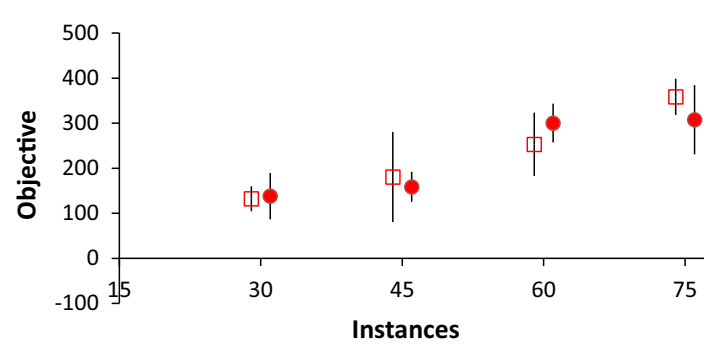

(d) Sample Size 200

Fig. 2. Computational results for the FSS and stochastic travel times, “ $\square$ " $n=2 m$, “○” $m=2 n$, deviations are small and lie within the marks.

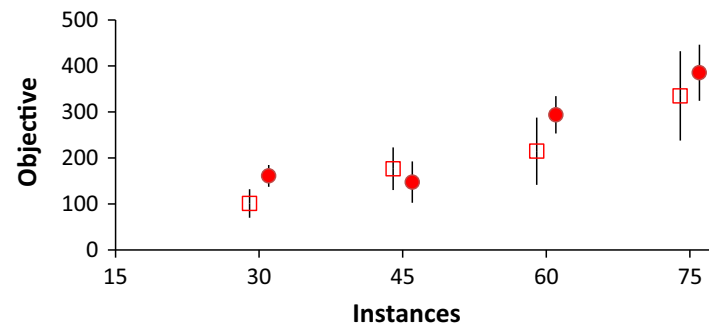

(a) Sample Size 10

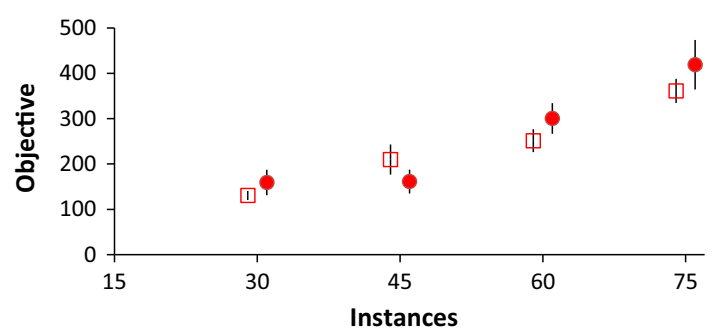

(c) Sample Size 100

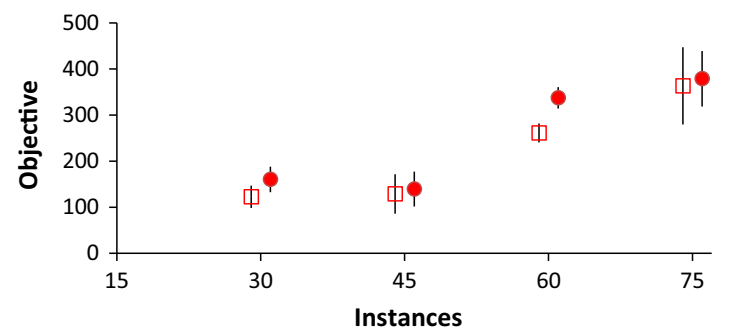

(b) Sample Size 50

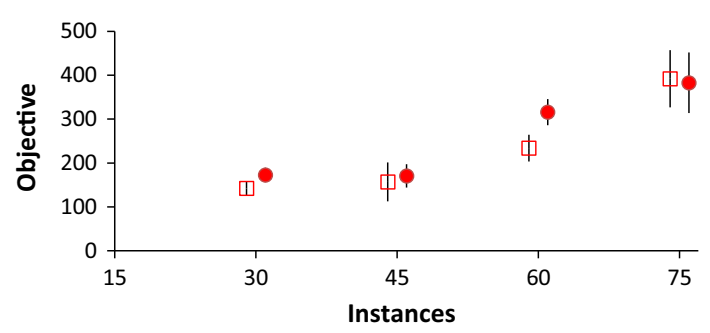

(d) Sample Size 200

Fig. 3. Computational results for the FSS and stochastic delivery locations, “ $\square$ " $n=2 m$, "O" $m=2 n$, deviations are small and lie within the marks.

Compared to the stochastic travel times, the results of stochastic delivery locations are quite stable. There is no significant difference in objective value between sample size 50 and 200, although the CPU time increases approximately proportionally to the sample size (see Table 3).

\subsection{Computational results of the $S A A$}

In Figs. 4 and 5, we present the approximate objective values and their standard deviations for different instances. The objective values are similar for different initial sample sizes. According to Table 4, the CPU time increases in most 
Table 3

CPU time (s) for the FSS.

\begin{tabular}{|c|c|c|c|c|c|c|c|c|}
\hline \multirow[t]{2}{*}{ Instances } & \multicolumn{4}{|c|}{ Stochastic travel times sample size } & \multicolumn{4}{|c|}{ Stochastic locations sample size } \\
\hline & 10 & 50 & 100 & 200 & 10 & 50 & 100 & 200 \\
\hline 30_1 & 46 & 206 & 197 & 496 & 51 & 149 & 259 & 693 \\
\hline 30_2 & 20 & 70 & 218 & 351 & 10 & 78 & 172 & 569 \\
\hline 45_1 & 46 & 330 & 505 & 833 & 44 & 530 & 633 & 1384 \\
\hline 45_2 & 22 & 220 & 232 & 675 & 31 & 215 & 445 & 1083 \\
\hline 60_1 & 84 & 525 & 972 & 1690 & 96 & 452 & 1129 & 1465 \\
\hline 60_2 & 49 & 392 & 713 & 1060 & 59 & 304 & 683 & 1548 \\
\hline 75_1 & 120 & 521 & 954 & 1892 & 112 & 889 & 1162 & 1774 \\
\hline 75_2 & 66 & 345 & 837 & 2244 & 69 & 443 & 1139 & 1868 \\
\hline
\end{tabular}

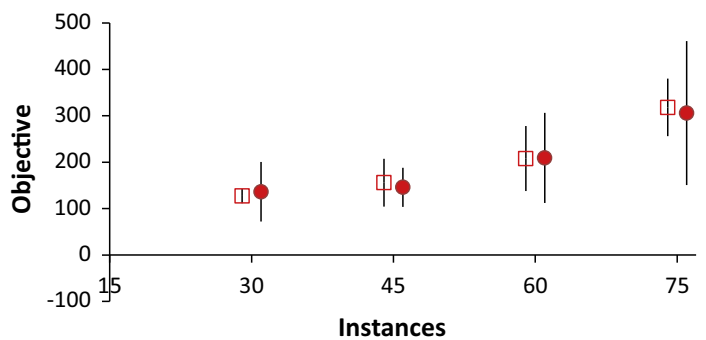

(a) Initial Sample Size 20

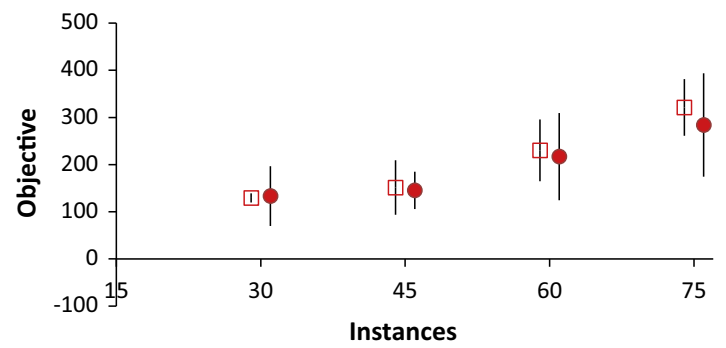

(b) Initial Sample Size 40

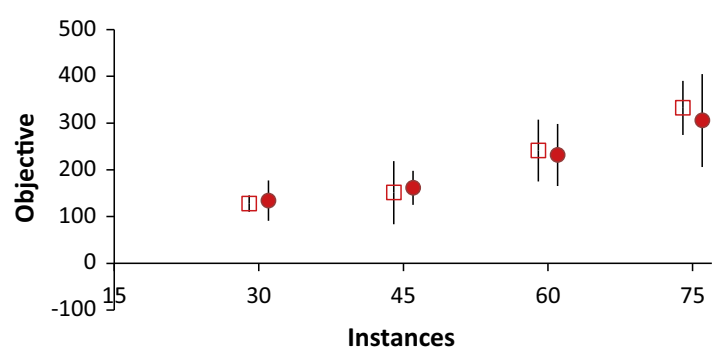

(c) Initial Sample Size 60

Fig. 4. Computational results for the SAA and stochastic travel times, “ $\square$ " $n=2 m$, “○” $m=2 n$, deviations are small and lie within the marks.

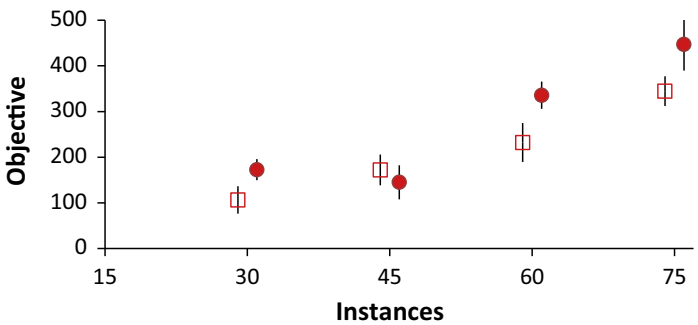

(a) Initial Sample Size 20

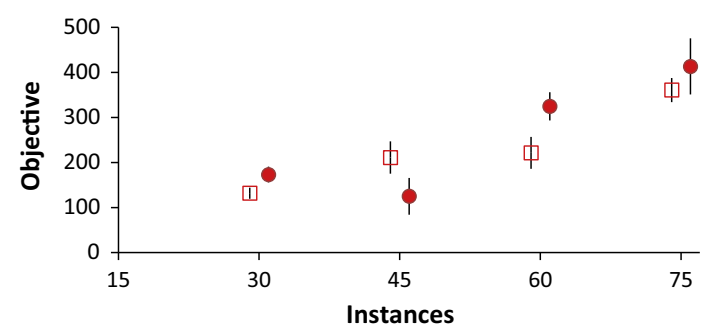

(b) Initial Sample Size 40

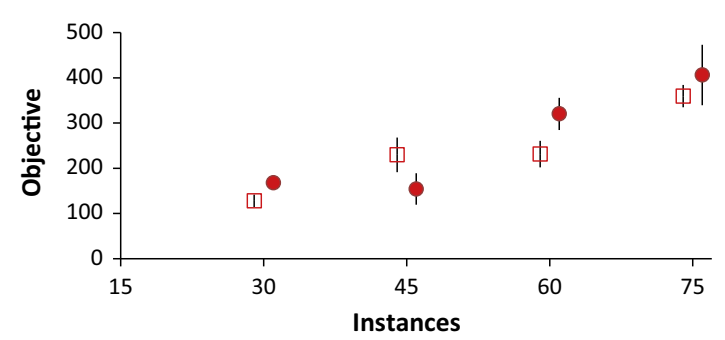

(c) Initial Sample Size 60

Fig. 5. Computational results for the SAA and stochastic delivery locations, “ $\square$ " $n=2 m$, “

$m=2 n$, deviations are small and lie within the marks. 
Table 4

CPU time (s) for SAA.

\begin{tabular}{|c|c|c|c|c|c|c|}
\hline \multirow[t]{2}{*}{ Instances } & \multicolumn{3}{|c|}{ Stochastic travel times: sample size } & \multicolumn{3}{|c|}{ Stochastic delivery locations: sample size } \\
\hline & 20 & 40 & 60 & 20 & 40 & 60 \\
\hline 30_1 & 277 & 130 & 311 & 83 & 94 & 372 \\
\hline 30_2 & 258 & 226 & 805 & 29 & 101 & 143 \\
\hline 45_1 & 566 & 943 & 915 & 165 & 350 & 729 \\
\hline 45_2 & 700 & 850 & 1825 & 96 & 279 & 1223 \\
\hline 60_1 & 944 & 1725 & 582 & 200 & 492 & 740 \\
\hline 60_2 & 1330 & 1856 & 1656 & 172 & 202 & 1328 \\
\hline 75_1 & 1296 & 1570 & 2915 & 288 & 733 & 1786 \\
\hline 75_2 & 1579 & 1629 & 2319 & 390 & 278 & 1700 \\
\hline
\end{tabular}

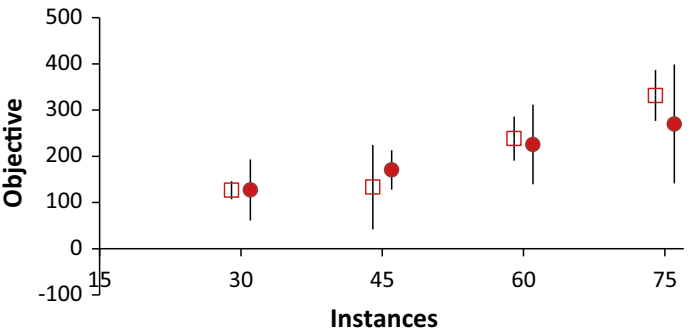

(a) Initial Sample Size 20

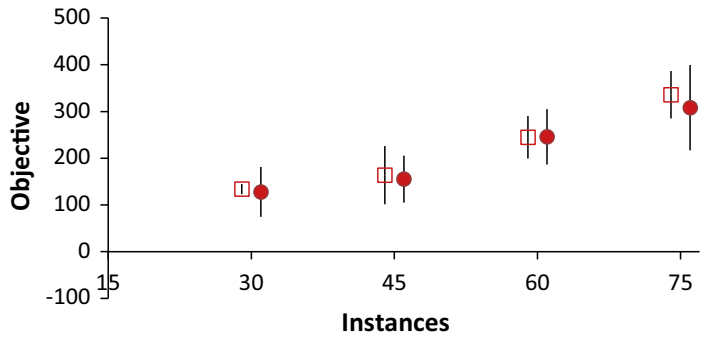

(b) Initial Sample Size 40

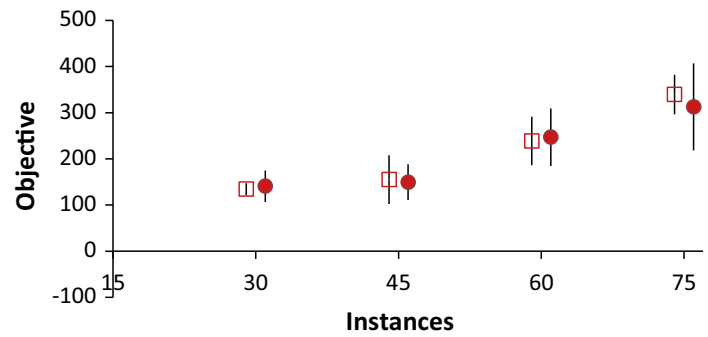

(c) Initial Sample Size 60

Fig. 6. Computational results for the SSP and stochastic travel times, “ $\square$ " $n=2 m$, "

circumstances. However, sometimes the CPU time decreases along with the increase of the sample sizes, e.g. the running time with 20 initial sample size is shorter than 40 initial sample size for instances 30_1 and 30_2. The reason is that if we set the sample size large enough, the algorithm converges faster. We learn from Fig. 5 that the objective values of stochastic delivery are quite stable. Another conclusion is that if the number of passengers is twice that of parcels, the objective value is much more sensitive than when the number of parcels exceeds that of passengers.

\subsection{Computational results of the SSP}

Figs. 6 and 7 show the result of the SSP, the interval of every point equals (mean - std.dev., mean + std.dev.). Overall, we obtained similar objective values in comparison with the FSS and the SAA. According to Table 5, the CPU time increases approximately by a factor of 3 as the sample size increases from 20 to 60 . When checking the details of Fig. 6, we found that the solution of $n_{k}=60$ is slightly better than $n_{k}=20$ and $n_{k}=40$ based on its values of the mean and standard deviation. For stochastic delivery, Fig. 7 shows that the solution of $n_{k}=20$ is as stable as the other two settings.

\subsection{Comparisons of the solutions of the proposed approaches}

The sampling size is fixed for the FSS. Moreover, only the initial sampling size is fixed in the SAA and SSP. The sampling size of the SAA and SSP increases based upon the performance of the previous iteration, and stops when the stopping criterion is reached. To make a comparison of the three methods, we presented the results with the similar CPU time for different approaches in this section. 


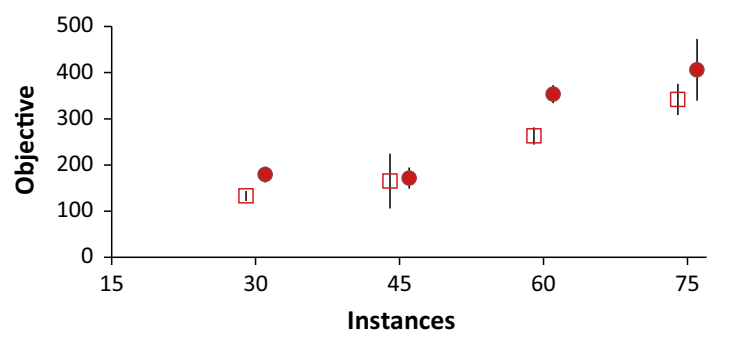

(a) Initial Sample Size 20

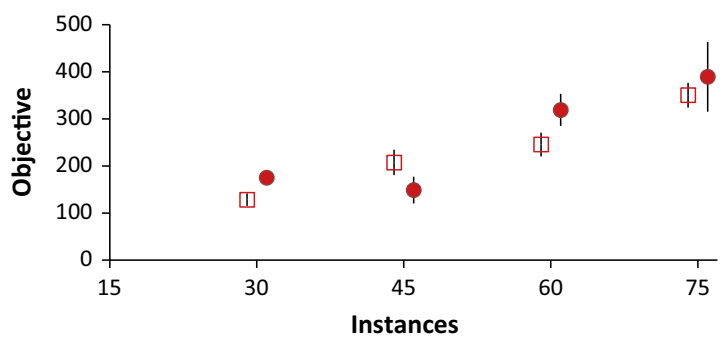

(b) Initial Sample Size 40

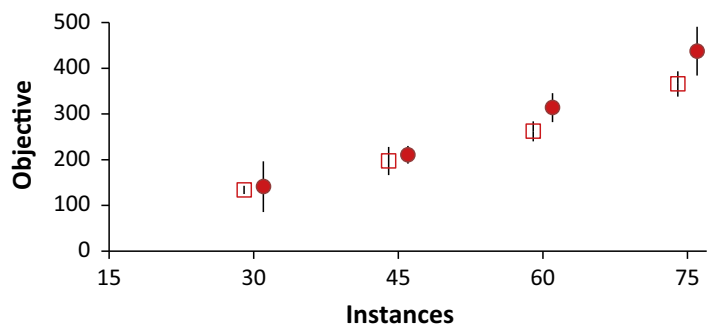

(c) Initial Sample Size 60

Fig. 7. Computational results for the SSP and stochastic delivery locations, “ $\square$ " $n=2 m$, “ $\bullet m=2 n$, deviations are small and lie within the marks.

Table 5

CPU time (s) for the SSP.

\begin{tabular}{|c|c|c|c|c|c|c|}
\hline \multirow[t]{2}{*}{ Instances } & \multicolumn{3}{|c|}{ stochastic travel times: sample size } & \multicolumn{3}{|c|}{ stochastic delivery locations: sample size } \\
\hline & 20 & 40 & 60 & 20 & 40 & 60 \\
\hline 30_1 & 196 & 319 & 376 & 832 & 1207 & 842 \\
\hline 30_2 & 101 & 163 & 287 & 173 & 324 & 1597 \\
\hline 45_1 & 557 & 827 & 1159 & 612 & 1348 & 1173 \\
\hline $45 \_2$ & 280 & 567 & 622 & 339 & 434 & 503 \\
\hline 60_1 & 1696 & 1299 & 1633 & 813 & 1349 & 1617 \\
\hline 60_2 & 1161 & 916 & 1064 & 246 & 978 & 1711 \\
\hline $75 \_1$ & 1057 & 1382 & 1923 & 1072 & 1992 & 2326 \\
\hline 75_2 & 1984 & 1261 & 1767 & 1126 & 771 & 2475 \\
\hline
\end{tabular}

Table 6

Stochastic travel times: Solution quality.

\begin{tabular}{lrlll}
\hline Instances & Deterministic & FSS & SAA & SSP \\
\hline 30_1 & $95.59 \pm 49.21$ & $133.04 \pm 8.74$ & $126.98 \pm 15.42$ & $133.86 \pm 10.81$ \\
30_2 & $90.38 \pm 110.73$ & $130.72 \pm 39.18$ & $136.31 \pm 64.21$ & $127.93 \pm 53.42$ \\
$45 \_1$ & $-427.27 \pm 399.50$ & $161.82 \pm 65.63$ & $155.79 \pm 51.54$ & $163.80 \pm 62.33$ \\
$45 \_2$ & $-70.94 \pm 156.34$ & $163.22 \pm 28.62$ & $145.78 \pm 42.14$ & $155.25 \pm 50.18$ \\
$60 \_1$ & $40.80 \pm 193.41$ & $242.27 \pm 33.76$ & $207.87 \pm 70.08$ & $244.77 \pm 45.41$ \\
60_2 & $135.76 \pm 295.30$ & $245.23 \pm 59.17$ & $209.21 \pm 97.11$ & $318.12 \pm 62.01$ \\
$75 \_1$ & $233.04 \pm 106.91$ & $353.50 \pm 40.20$ & $305.86 \pm 155.15$ & $35.87 \pm 50.72$ \\
$75 \_2$ & $-294.35 \pm 363.38$ & $343.08 \pm 73.53$ & & $308.17 \pm 91.29$ \\
\hline
\end{tabular}

Table 7

Stochastic delivery locations: Solution quality.

\begin{tabular}{lclll}
\hline Instances & Deterministic & FSS & SAA & SSP \\
\hline 30_1 & $66.85 \pm 66.10$ & $130.58 \pm 9.92$ & $106.34 \pm 30.03$ & $128.44 \pm 13.25$ \\
30_2 & $30.69 \pm 52.55$ & $159.26 \pm 28.08$ & $172.74 \pm 23.12$ & $172.10 \pm 33.67$ \\
$45 \_1$ & $78.82 \pm 120.90$ & $209.83 \pm 33.19$ & $144.94 \pm 37.28$ & $207.46 \pm 26.93$ \\
$45 \_2$ & $50.54 \pm 29.69$ & $161.20 \pm 26.27$ & $232.11 \pm 42.68$ & $148.71 \pm 28.43$ \\
$60 \_1$ & $31.96 \pm 120.86$ & $251.47 \pm 25.25$ & $335.74 \pm 29.86$ & $245.45 \pm 25.28$ \\
$60 \_2$ & $100.91 \pm 66.58$ & $300.36 \pm 33.80$ & $344.61 \pm 32.59$ & $319.10 \pm 33.94$ \\
$75 \_1$ & $341.59 \pm 96.70$ & $361.28 \pm 26.61$ & $451.88 \pm 57.24$ & $350.19 \pm 26.33$ \\
$75 \_2$ & $46.04 \pm 96.22$ & $419.00 \pm 54.63$ & & $389.20 \pm 73.95$ \\
\hline
\end{tabular}


Table 8

Value of stochastic solution.

\begin{tabular}{|c|c|c|c|c|}
\hline & VSS of the ST & VSS of the SD & ST $\%$ & SD \% \\
\hline 30_1 & 36.63 & 75.30 & 38.32 & 112.63 \\
\hline 30_2 & 47.64 & 142.09 & 52.72 & 463.05 \\
\hline 45_1 & 607.75 & 78.15 & - & 99.15 \\
\hline 45_2 & 229.64 & 120.10 & - & 237.63 \\
\hline 60_1 & 212.28 & 201.97 & 520.31 & 632.04 \\
\hline 60_2 & 436.07 & 215.00 & - & 213.07 \\
\hline 75_1 & 125.41 & 50.47 & 53.82 & 14.78 \\
\hline 75_2 & 611.94 & 336.88 & - & 731.66 \\
\hline
\end{tabular}

-: No feasible result because the expected value of the deterministic solution is negative.

Tables 6 and 7 show the obtained objective values. The first column denotes the instances. The columns "Deterministic", "FSS","SAA", "SSP" refer to the objective value obtained from the following methods: the deterministic solution, the FSS, the SAA, and the SSP. For the last three approaches, we intentionally present the objective value obtained by different approaches with approximately the same CPU time. For stochastic delivery locations, there is no significant difference, but the column "FSS" provides the best results for stochastic travel times. Moreover, the FSS can always get a relatively reliable solution which is independent from initial sample size, compared to the SAA and the SSP on all the testing (see Figs. 27). Finally, the comparisons between the stochastic and deterministic solution confirm the benefit and effectiveness of the stochastic approach. The profit of stochastic solutions is higher and more stable than deterministic solutions (deviations of the FSS, the SAA and the SSP are much smaller than those of deterministic solutions tested against a set of scenarios), which can be seen from Tables 6 and 7 .

In order to provide an impression of the value of our computation, we present the value of the stochastic solution in Table 8. The value of the stochastic solution (VSS) is normally used to measure the value of implementing a stochastic model. The VSS equals to the potential benefit from solving the stochastic program minus solving a deterministic program, for the related definition of VSS we refer to Birge (1982). The FSS with sample size 200 as the benefit from solving the stochastic program are used for comparison. The first column represents the instances. The second and third column ("VSS of the ST" and "VSS of the SD") are the VSS for stochastic travel times and delivery locations, separately. The percentage of improvement (VSS divides the expected result of the deterministic solution) are shown under the columns "ST\%" and "SD\%". From Table 8 one can see the stochastic information is valuable to improve the profit for both stochastic delivery time and delivery locations.

\section{Conclusion}

In this paper, we modeled the SARP as a stochastic program with recourse; we focused on realistic objective functions and constraints that contain uncertainty. We implemented an adaptive large neighborhood search heuristic, and provided a numerical study of the SARP with stochastic travel times and delivery locations. The computational results suggest that the fixed sample size of 200 realizations provides a good approximation to the formulated scenario, by a comparison of the proposed approaches (deterministic, the FSS, the SAA, and the SSP). The SAA and the SSP approaches increase the scenario size adaptively. We learned that the SARP is more sensitive to the stochastic travel times than the stochastic delivery locations. The explanation is that the stochastic travel time is affected by the road congestion, which is fluctuated in a wide range. Compare to stochastic travel time, the stochatility of delivery location is not obvious in our setting. Though the CPU time of the stochastic SARP is increased compared to the deterministic SARP, the objective value of the stochastic SARP significantly increases and the results are much more stable than those of the deterministic SARP.

The comparison of the stochastic and deterministic solution has been presented. The results suggest that it is desirable to analyze the stochastic characteristics of requests before implementing a taxi sharing service. The reason is that the profit of stochastic solutions is much higher than that of deterministic solutions tested against stochastic scenarios.

In the future, one promising research venue is to implement decomposition techniques to reduce the CPU time. In order to find a guaranteed optimal solution, one should develop exact algorithms though the CPU time may increase. In this paper, two sources of stochasticity are considered separately. Another possible direction is designing an efficient approach for handling several sources of stochasticity within a single model. 


\section{Appendix A}

\section{Procedure 1.}

Input: Initial sample size $N$ and $N^{\prime}\left(N^{\prime} \gg N\right)$

Output: Candidate solution, $\hat{x}$

1. Determine the number $M$ for the SAA replications.

2. For $m=1, \ldots, M$, do Step 2.1 through 2.2:

2.1 Generate a sample of size $N$ and solve the SAA problem with objective value $\hat{v}_{N}^{m}$ and solution $\hat{x}_{N}^{m}$. In the $m$-th iteration, we apply the ALNS to solve the problem, and choose a best history solution $x_{i}^{*}:=\underset{i \in\{1, \ldots, m\}}{\arg \min } 1 / N^{\prime} \sum_{j=1}^{N^{\prime}} G\left(x_{i}, W^{j}\right)$ as the initial solution.

2.2 Generate a sample of size $N^{\prime}$ to estimate the optimality gap and the variance of the gap estimator.

If the optimality gap and the variance of the gap estimator are sufficiently small (explanation to follow), go to Step 4.

3. If the optimal gap or the variance of the gap estimator is too large (explanation to follow), increase the sample size $N$ and/or $N^{\prime}$, and return to Step 2 .

4. Choose the best solution $\hat{x}$ among all candidate solutions $\hat{x}_{N}^{m}$ produced, using a screening and selection procedure (Kleywegt et al. Kleywegt et al. (2001)). Stop.

In Step 2.2, if $M$ replications have been tested, let $\hat{g}_{N^{\prime}}(\hat{x})$ and $\bar{v}_{N}^{M}$ stand for the estimated upper and lower bound of the gap, respectively. The estimated gap equals $\hat{g}_{N^{\prime}}(\hat{x})-\bar{v}_{N}^{M}$, and variance is $\left(\frac{S_{N^{\prime}}^{2}(\hat{x})}{N^{\prime}}+\frac{S_{M}^{2}}{M}\right)^{1 / 2}$. The confidence interval of the estimated gap is: $\left[\hat{g}_{N^{\prime}}(\hat{x})-\bar{v}_{N}^{M}-z_{\alpha}\left(\frac{s_{N^{\prime}}^{2}(\hat{x})}{N^{\prime}}+\frac{S_{M}^{2}}{M}\right)^{1 / 2}, \hat{g}_{N^{\prime}}(\hat{x})-\bar{v}_{N}^{M}+z_{\alpha}\left(\frac{s_{N^{\prime}}^{2}(\hat{x})}{N^{\prime}}+\frac{S_{M}^{2}}{M}\right)^{1 / 2}\right]$.

In Step 2.3, we define "sufficiently small" as: gap is a gap at iteration t, and $\mid\left(\right.$ gap $_{t-1}-$ gap $\left._{t}\right) \mid /$ gap $_{t}$ is less than $5 \%$, or the upper bound of the confidence interval of the gap $/ \bar{v}_{N}^{M}$ is less than $10 \%$. For the proof of convergence properties of the SAA, we refer to Kleywegt et al. Kleywegt et al. (2001).

The equations for calculating $\hat{g}_{N^{\prime}}(\hat{x}), \hat{g}_{N^{\prime}}(\hat{x}), S_{N^{\prime}}^{2}(\hat{x}) / N^{\prime}, S_{M^{\prime}}^{2} / M^{\prime}$ are as follows:

$$
\begin{aligned}
& \hat{g}_{N^{\prime}}(\hat{x})=\frac{1}{N^{\prime}} \sum_{j=1}^{N^{\prime}} G\left(\hat{x}, W^{j}\right) \\
& \bar{v}_{N}^{M^{\prime}}=\frac{1}{M^{\prime}} \sum_{m=1}^{M^{\prime}} \hat{v}_{N}^{m} \\
& S_{N^{\prime}}^{2}(\hat{x}) / N^{\prime}=\frac{1}{N^{\prime}\left(N^{\prime}-1\right)} \sum_{m=1}^{N^{\prime}}\left(\hat{v}_{m}(\hat{x})-\bar{v}_{N^{\prime}}(\hat{x})\right)^{2} \\
& S_{M^{\prime}}^{2} / M^{\prime}=\frac{1}{M^{\prime}\left(M^{\prime}-1\right)} \sum_{m=1}^{M^{\prime}}\left(\hat{v}_{N}^{m}-\bar{v}_{N}^{M^{\prime}}\right)^{2}
\end{aligned}
$$

\section{Procedure 2.}

Input: Values for $h>h^{\prime}>0, \epsilon>\epsilon^{\prime}>0,0<\alpha_{\text {sto }}<1, p>0$, and resampling frequency $k_{f}$ (a positive integer)

Output: Candidate solution, $\hat{x}_{T}$

1. Set $k:=1$, calculate $n_{k}$, and sampling observations $\tilde{\xi}^{1}, \tilde{\xi}^{2}, \ldots, \tilde{\xi}^{n_{k}}$.

2. Use $\tilde{\xi}^{1}, \tilde{\xi}^{2}, \ldots, \tilde{\xi}^{n_{k}}$ to form $G_{k}$ and $s_{k}^{2}$. When applying the ALNS to solve the problem, we use a screening and selection procedure (Kleywegt et al., 2001) to choose a best solution as the initial solution.

3. If $G_{k} \leqslant h^{\prime} s_{k}+\epsilon^{\prime}$, then set $T:=k$, and go to Step 5 .

4. Set $k:=k+1$ and calculate $n_{k}$. If $k_{f}$ divides $k$, then sample observation $\tilde{\xi}^{1}, \tilde{\xi}^{2}, \ldots, \tilde{\xi}^{n}$, independently of samples generated in previous iterations. Else, sample $n_{k}-n_{k-1}$ observations $\tilde{\xi}^{n_{k-1}+1}, \tilde{\xi}^{n_{k-1}+2}, \ldots, \tilde{\xi}^{n_{k}}$ from the distribution of $\tilde{\xi}$. Go to Step 2.

5. Output candidate solution $\hat{x}_{T}$.

The equations for calculating $n_{k}, G_{k}, s_{k}^{2}$ are as follows: 


$$
\begin{aligned}
n_{k} & =\left\lceil\left(\frac{1}{h-h^{\prime}}\right)^{2}\left(\max \left\{2 \ln \sum_{j=1}^{\infty} j^{-p l n j} / \sqrt{2 \pi} \alpha_{s t o}, 1\right\}+2 p \ln ^{2} k\right)\right\rceil \\
G_{k} & =\frac{1}{n_{k}} \sum_{i=1}^{n_{k}}\left(f\left(x_{k}, \tilde{\epsilon}^{i}\right)-f\left(x_{n_{k}}, \tilde{\epsilon}^{i}\right)\right) \\
s_{k}^{2} & =\frac{1}{n_{k}-1} \sum_{i=1}^{n_{k}}\left[\left(f\left(x_{k}, \tilde{\epsilon}^{i}\right)-f\left(x_{n_{k}}, \tilde{\epsilon}^{i}\right)\right)-G_{k}\right]^{2}
\end{aligned}
$$

\section{References}

Bayraksan, G., Morton, D., 2011. A sequential sampling procedure for stochastic programming. SIAM J. Optimiz. 59 (4), $898-913$.

Bianchi, L., Birattari, M., Chiarandini, M., Manfrin, M., Mastrolilli, M., Paquete, L., Rossi-Doria, O., Schiavinotto, T., 2006. Hybrid metaheuristics for the vehicle routing problem with stochastic demands. J. Math. Model. Algor. 5 (1), 91-110.

Birge, J.R., 1982. The value of the stochastic solution in stochastic linear programs with fixed recourse. Math. Program. 24 (1), $314-325$.

Christiansen, C.H., Lysgaard, J., 2007. A branch-and-price algorithm for the capacitated vehicle routing problem with stochastic demands. Operat. Res. Lett. 35 (6), 773-781.

Cordeau, J.F., Laporte, G., 2003. A tabu search heuristic for the static multi-vehicle dial-a-ride problem. Transport. Res. Part B 37 (6), $579-594$.

Dantzig, G., 1955. Linear programming under uncertainty. Manage. Sci. 1, 197-206.

Dupačová, J., Gröwe-Kuska, N., Römisch, W., 2003. Scenario reduction in stochastic programming. Math. Program. 95 (3), $493-511$.

Fagnant, D.J., Kockelman, K.M., 2014. The travel and environmental implications of shared autonomous vehicles, using agent-based model scenarios. Transport. Res. Part C: Emerg. Technol. 40, 1-13.

Fu, L., 2002. Scheduling dial-a-ride paratransit under time-varying, stochastic congestion. Transport. Res. Part B: Methodol. 36 (6), $485-506$.

Gauvin, C., Desaulniers, G., Gendreau, M., 2014. A branch-cut-and-price algorithm for the vehicle routing problem with stochastic demands. Comput. Operat. Res. 50, 141-153.

Gendreau, M., Guertin, F., Potvin, J.Y., Séguin, R., 2006. Neighborhood search heuristics for a dynamic vehicle dispatching problem with pick-ups and deliveries. Transport. Res. Part C: Emerg. Technol. 14 (3), 157-174.

Gutjahr, W., 2011. Recent trends in metaheuristics for stochastic combinatorial optimization. Central Euro. J. Comput. Sci. 1 (1), 58-66.

Heilporn, G., Cordeau, J.F., Laporte, G., 2011. An integer L-shaped algorithm for the dial-a-ride problem with stochastic customer delays. Discr. Appl. Math. 159 (9), 883-895.

Hu, Z.H., Sheu, J.B., Zhao, L., Lu, C.C., 2015. A dynamic closed-loop vehicle routing problem with uncertainty and incompatible goods. Transport. Res. Part C: Emerg. Technol. 55, 273-297.

King, A.J., Wallace, S.W., 2012. Modeling with Stochastic Programming. Springer.

Kleywegt, A., Shapiro, A., Homem-De-Mello, T., 2001. The sample average approximation method for stochastic discrete optimization. SIAM J. Optimiz. 12 (2), 479-502.

Li, B., Krushinsky, D., Reijers, H.A., Van Woensel, T., 2014. The share-a-ride problem: people and parcels sharing taxis. Euro. J. Operat. Res. 238 (1), $31-40$.

Li, B., Krushinsky, D., Van Woensel, T., Reijers, H.A., 2016. An adaptive large neighborhood search heuristic for the share-a-ride problem. Comput. Operat. Res. 66, 170-180.

Marković, N., Nair, R., Schonfeld, P., Miller-Hooks, E., Mohebbi, M., 2015. Optimizing dial-a-ride services in Maryland: benefits of computerized routing and scheduling. Transport. Res. Part C: Emerg. Technol. 55, 156-165.

Muelas, S., LaTorre, A., Peña, J.M., 2015. A distributed VNS algorithm for optimizing dial-a-ride problems in large-scale scenarios. Transport. Res. Part C: Emerg. Technol. 54, 110-130.

Ropke, S., Pisinger, D., 2006. An adaptive large neighborhood search heuristic for the pickup and delivery problem with time windows. Transport. Sci. 40 (4), 455-472.

Schilde, M., Doerner, K.F., Hartl, R.F., 2011. Metaheuristics for the dynamic stochastic dial-a-ride problem with expected return transports. Comput. Operat. Res. 38 (12), 1719-1730.

Wang, C., Handoko, S.D., Lau, H.C., 2014. An auction with rolling horizon for urban consolidation centre. In: 2014 IEEE International Conference on Service Operations and Logistics, and Informatics (SOLI). IEEE, pp. 438-443. 